\title{
Un Estudio sobre las Metas Académicas en Estudiantes Universitarios de Enfermería
}

\section{A Study of the Academic Goals of College Nursing Sudents}

\author{
Ana Navea Martín \\ Universidad Alfonso X El Sabio, España
}

\begin{abstract}
Resumen. El objetivo de la investigación consistió en conocer las principales características de las metas académicas, en una muestra universitaria que cursa $2^{\circ}$ en los estudios de la Diplomatura de Enfermería en una universidad privada. El instrumento utilizado en la investigación fue el cuestionario de metas de Skaalvik (1997). Este instrumento fue validado en el estudio y además se obtuvieron tres grupos de estudiantes en función de la adopción de múltiples metas por parte de éstos. Esta investigación aporta datos que refuerzan la estructura de cuatro metas (meta de tarea, meta de autoensalzamiento, meta de autofrustración y meta de evitación del trabajo) establecidas por Skaalvik en 1997, obtiene datos acerca de la correlación positiva entre la meta de tarea y la meta de autoensalzamiento y clasifica a los estudiantes en función a las metas hacia las que se orientan, predominando la orientación hacia la meta de tarea frente a las demás metas. Estos Resultados concuerdan con aspectos vocacionales que se han relacionado con la elección de carreras de ciencias de la salud, especialmente de enfermería. Para finalizar se ofrecen propuestas de mejora para la intervención en la práctica educativa.

Palabras clave: motivación académica, metas académicas, múltiples metas, aspectos vocacionales, aprendizaje autorregulado.
\end{abstract}

\begin{abstract}
The objective of this study was to understand the main features of the academic goals shown by a sample of students in their second year of studies for the nursing diploma at a private university. The instrument used was the goals questionnaire by Skaalvik (1997). This instrument was validated in the study, and obtained three groups of students based on their adoption of multiple goals. This study provides data that reinforce the four-goal structure (target task, self-enchancing goal, self-defeating goal, and work avoidance goal) set out by Skaalvik in 1997. This study also collects data about the positive correlation between the task and self-enhancing goals, and classifies students according to the goals towards which they are oriented, with an orientation toward the goal orientation task predominating over the others.

These results are consistent with vocational aspects that are related to the choice of degrees in health sciences, especially nursing. Finally, suggestions for improvement are offered for intervention in educational practice.

Keywords: academic motivation, academic goals, multiple goals, vocational aspects, self-regulated learning.
\end{abstract}

\section{Introducción}

Actualmente existe un interés creciente entre los teóricos de la educación acerca de la motivación académica, su evaluación y la intervención en los procesos que implica.

El aspecto fundamental que trata de explicar el concepto de motivación es la causa de las conductas concretas. ¿Por qué un alumno se esfuerza por estudiar y otros alumnos prefieren divertirse con sus amigos?

Tomando como referencia la definición clásica de motivación, podemos considerarla como un conjunto de procesos implicados en la activación, dirección y persistencia de la conducta. Según Beltrán (1998) algunos alumnos se entusiasman con facilidad pero

La correspondencia sobre este artículo debe enviarse a la autora al e-mail: ana.navea@salud.madrid.org ceden rápidamente, se activan pero realmente no persisten; otros se activan y persisten pero en una dirección equivocada; finalmente, tenemos estudiantes que funcionan muy bien en los tres niveles, se entusiasman fácilmente por algo, persisten y, además, saben caminar en la dirección adecuada.

Dentro de la motivación académica, podemos diferenciar tres componentes o dimensiones fundamentales. La primera dimensión tiene que ver con los motivos, propósitos o razones para implicarse en la realización de una actividad. Estos aspectos están englobados dentro de lo que es el componente motivacional de valor, ya que la mayor o menor importancia y relevancia que una persona le asigna a la realización de una actividad es lo que determina, en este caso, que la lleve a cabo o no.

Una segunda dimensión de la motivación académica es denominada componente de expectativa, y 
engloba las percepciones y creencias individuales sobre la capacidad para realizar una tarea. Si el estudiante no está convencido de que tiene la capacidad y la competencia necesaria para realizar la tarea, aunque tenga fuertes motivos para hacerla, no se movilizará la conducta.

Un tercer componente lo constituyen las variables afectivas o emocionales, que comparten con los procesos motivacionales los siguientes aspectos (González-Pienda, González, Nuñez y Valle, 2002):

- Ambos son procesos funcionales, es decir permiten a las personas adaptarse al ambiente.

- Están estrechamente interrelacionados, en el sentido de que la consecución de metas para las que está motivada una persona genera reacciones emocionales positivas y la no consecución de esas metas produce reacciones negativas.

- Ambos procesos (motivación y emoción) mantienen relaciones importantes con otros procesos psicológicos como la percepción, la memoria y el aprendizaje.

En resumen, la motivación requiere, por tanto un cierto equilibrio personal entre las creencias de competencia, el interés personal y las emociones que nos provocan. Dado que todas las actividades de logro son más o menos difíciles, sólo se dedicará tiempo y esfuerzo, si se consigue un balance positivo entre las emociones y las consideraciones personales.

Dentro de los procesos motivacionales, se establece que la conducta siempre está orientada por metas, las cuales desempeñan un importante papel en el proceso motivacional, definiendo su contenido y dirección (Suárez y Fernández, 2004). Sobre las metas, se han realizado muchas investigaciones sobre todo relacionadas con las posibles clasificaciones o los tipos de metas. Skaalvik, (1997), propone cuatro tipos de metas académicas:

- Meta de orientación a la tarea o meta de tarea, en la que predomina "el querer aprender", sin pensar en recompensas externas.

- Dos metas orientadas al yo o de rendimiento: la meta de ego orientación a la mejora o meta de autoensalzamiento, en la que la persona quiere demostrar sus capacidades ante los demás y la meta de ego orientación a la frustración o meta de autofrustración, en la que la persona está interesada en no ser juzgada negativamente por los demás.

- La última meta es la meta de evitación del trabajo, donde el objetivo es hacer las tareas con el menor esfuerzo posible.

Las metas académicas han sido de gran importancia para la explicación de la motivación y conducta de los estudiantes. Tradicionalmente las teorías acerca de las metas establecían dos tipos de metas que se consideraban mutuamente excluyentes, las metas de aprendizaje y las metas de rendimiento, sin embargo una nueva perspectiva, considera las múltiples metas como un aspecto importante del aprendizaje autorregulado, es decir los estudiantes pueden perseguir más de una meta dentro de su proceso de aprendizaje (Suárez, González, Abalde, Valle, Rodríguez y Piñeiro 2001).

En un estudio de Suárez, González y Valle (2001), realizado con muestra universitaria en España, los alumnos se estructuraron en tres grupos en función a la orientación de sus metas.

En el primer grupo predominaban los estudiantes con alta meta de ego orientación a la mejora (selfenhancing), alta meta de orientación a la frustración (self-defeating), alta meta de evitación del trabajo y unos valores medios de meta de tarea. En el segundo grupo predominaban los estudiantes con alta meta de tarea y alta meta de ego orientación a la frustración y valores medios de meta de ego de orientación a la mejora y meta de evitación al trabajo. Por último el tercer grupo obtenido en esta investigación lo formaron los estudiantes con alta meta de tarea, valores medios de meta de evitación al trabajo y valores bajos de meta de ego orientación a la mejora y meta de ego orientación a la frustración. Los resultados de esta investigación por tanto, nos indican la posibilidad de la adopción de múltiples metas a la hora de realizar los aprendizajes.

En otro estudio de Suárez, González, Abalde, Valle, Rodríguez y Piñeiro (2001), las metas académicas fueron medidas a través de las escalas de orientación a metas elaboradas por Skaalvik (1997), que distingue cuatro tipos, una meta de orientación a la tarea, dos metas englobadas dentro de las metas del yo, la meta de autoensalzamiento del ego y la meta de autofrustración del ego y una última meta de evitación del trabajo. Los estudiantes que emplearon múltiples metas, en la que predominaba una meta de tarea y una meta de rendimiento, siendo ésta una meta de autofrustración del ego, se caracterizaban por emplear un aprendizaje más estratégico. Así pues, para los estudiantes no sólo es importante aprender por el aprendizaje en sí mismo, sino también hacerlo sabiendo que reciben apreciaciones positivas de los demás.

En resumen, los determinantes de las metas académicas incluyen tanto las características motivacionales del alumno como los aspectos instruccionales y contextuales. Entre los distintos factores personales que se han estudiado en relación con la motivación se pueden señalar las atribuciones, la autoeficacia, la percepción de la autocompetencia, el interés...

En este trabajo se van a estudiar los distintos tipos de metas y la adopción de múltiples metas por parte de los estudiantes universitarios de enfermería.

\section{Diseño de la investigación}

\section{Objetivos}

- Conocer las principales características de las metas académicas, en una muestra universita- 
ria que cursa $2^{\circ}$ curso en los estudios de la Diplomatura de Enfermería en una universidad privada.

- Estudiar la fiabilidad y la validez de constructo del instrumento utilizado.

- Describir el tipo de metas predominantes utilizadas por los estudiantes.

- Conocer la tipología de los estudiantes en función de sus metas.

\section{Metodología}

Para la realización de la presente investigación se ha seleccionado un grupo de estudiantes universitarios, los cuales tienen unas características ya valoradas en otros estudios como una mayor gestión de su propio aprendizaje, establecimiento de metas, utilización de estrategias y en definitiva un mayor aprendizaje autorregulado.

La investigación tiene un carácter sobre todo descriptivo centrado en la validación del instrumento, el cuestionario de Skaalvik (1997) y en el análisis de las variables también con respecto a la adopción de múltiples metas.

\section{Variables}

El estudiante durante su aprendizaje puede optar por la orientación hacia metas de aprendizaje, o bien por la orientación a metas de rendimiento. Estas metas, según Skaalvik, se pueden dividir a su vez en metas de autoensalzamiento del ego y en metas de autofrustración del ego. Es decir, el estudiante puede querer obtener unos buenos resultados a fin de demostrar su gran capacidad o para evitar los juicios negativos de los demás. Por último habría una cuarta meta, que consistiría en realizar el trabajo con el menor esfuerzo posible, que este autor denominó meta de evitación del trabajo. Estos cuatro tipos de metas establecidas por Skaalvik (1997), constituyen las variables objeto del estudio. En resumen, las variables que se van a estudiar son:

- La meta de tarea.

- La meta de autoensalzamiento del ego.

- La meta de autofrustración del ego.

- La meta de evitación del trabajo.

\section{Población y muestra}

La muestra está formada por 103 estudiantes de $2^{\circ}$ curso de la Diplomatura de Enfermería de la Universidad privada Alfonso X El Sabio, cuyo campus se encuentra situado en Villanueva de la Cañada (Madrid). De la muestra 16 son hombres y 87 son mujeres, con edades comprendidas entre los 20 y los 30 años. La muestra fue escogida entre los estudiantes que asisten a clases presenciales de la asignatura de Enfermería en Salud Mental, de cuatro grupos, dos de mañana y dos de tarde.

\section{Técnicas e instrumentos de recogida de información}

El instrumento utilizado para la recogida de datos es el Cuestionario de Metas de Skaalvik (1997).El cuestionario ha sido adaptado a la muestra del estudio.

\section{Análisis de datos}

\section{Procedimiento de recogida de los datos.}

La aplicación de las pruebas fue realizada en un único momento temporal por separado en cada uno de los grupos (2202, 2203, 2204 y 2205), cada grupo en su aula, dos grupos durante la mañana y dos grupos durante la tarde.

\section{Técnicas de análisis de datos.}

En primer lugar, se procedió a la realización de un análisis factorial del cuestionario para ver su estructura, mediante el análisis por componentes principales y la rotación varimax. Se especificó el número de factores (4), para comprobar si nuestros resultados se ajustaban a investigaciones anteriores que establecen en el análisis factorial la existencia de cuatro factores en los que se agrupan los ítems del cuestionario, coincidiendo con los cuatro tipos de metas.

En segundo lugar se analizó la escala de metas, haciendo un análisis de fiabilidad, mediante de Cronbach del cuestionario en su totalidad.

A continuación se estudiaron a nivel descriptivo las medias y desviaciones típicas de cada meta y cómo correlacionan entre ellas.

Finalmente se realiza un análisis de cluster de las metas, con el objetivo de estudiar cómo se agrupan los estudiantes en función de su adopción de múltiples metas. En anteriores estudios, (Suárez, González, y Valle, 2001), se obtuvieron tres grupos de estudiantes, predominando en cada grupo la adopción de unas metas sobre otras.

Los análisis se realizaron con el programa SPSS15.0 versión para Windows.

\section{Resultados}

Tras la realización del análisis factorial se obtienen 4 factores que explican el $21.63 \%$ de la varianza total 
Tabla 1. Resultados obtenidos con el análisis factorial por componentes principales y rotación varimax

\begin{tabular}{cccc}
\hline Componente & Total & \% Varianza & \% Acumulado \\
\hline 1 & 3.46 & 21.63 & 21.63 \\
2 & 2.55 & 15.93 & 37.57 \\
3 & 2.07 & 12.98 & 50.56 \\
4 & 1.41 & 8.83 & 59.39 \\
\hline
\end{tabular}

Tabla 2. Resultados de la fiabilidad del cuestionario en su totalidad y de la fiabilidad de cada una de las metas de Skaalvik mediante el de Cronbach

\begin{tabular}{lc}
\hline & $\begin{array}{c}\text { Alfa de } \\
\text { Cronbach }\end{array}$ \\
\hline Fiabilidad del Test completo & 0.72 \\
Fiabilidad de la variable meta de tarea & 0.55 \\
Fiabilidad de la variable meta de autoensalzamiento del ego & 0.79 \\
Fiabilidad de la variable meta de autofrustración del ego & 0.90 \\
Fiabilidad de la variable meta de evitación del trabajo & 0.69 \\
\hline
\end{tabular}

(componente 1), el $15.93 \%$ de la varianza total (componente 2), el $12.98 \%$ de la varianza total (componente $^{\circ} 3$ ) y finalmente, el $8.83 \%$ de la varianza total (componente 4).

La estructura factorial una vez obtenida, es de cuatro factores coincidiendo, con las cuatro metas de Skaalvik (1997).

Para calcular la fiabilidad del test completo, como consistencia interna de la escala, se utiliza el estadístico de Cronbach, cuyo resultado obtenido fue de 0.72 .

La fiabilidad de la meta de tarea obtenida fue de 0.55 (la más baja), la fiabilidad de la meta de autoensalzamiento del ego fue de 0.79 , la fiabilidad de la meta de autofrustración del ego fue de 0.90 (la más alta) y por último la fiabilidad de la meta de evitación del trabajo fue de 0.69 .

Tras el análisis de correlación de Pearson entre las metas, según se observa en la Tabla 3, la correlación entre la meta de tarea y la meta de autoensalzamiento del ego es significativa al nivel 0.05 bilateral.

Según el análisis de cluster, la muestra se clasifica en tres grupos con aproximadamente el mismo número de participantes en cada uno, cuya diferencia fundamental consiste en los valores bajos o medios de las metas de autoensalzamiento, de autofrustración y de evitación.

- Cluster 1: Alta meta de tarea, alta meta de autoensalzamiento del ego y valores medios de autofrustración del ego y de evitación del trabajo. Lo componen 34 sujetos.

- Cluster 2: Alta meta de tarea, y bajas metas de autoensalzamiento del ego, autofrustración del ego y evitación del trabajo. Lo componen 31 sujetos

- Cluster 3: Alta meta de tarea y alta meta de autofrustración del ego, valores medios de meta de autoensalzamiento del ego y de meta de evitación del trabajo. Lo componen 37 sujetos.

Se observa que en la muestra todos los estudiantes dicen utilizar la meta de tarea con frecuencia.

\section{Conclusiones}

El cuestionario de metas de Skaalvik presenta una fiabilidad de 0.72 , por tanto aceptable, y cada meta tiene una fiabilidad que oscila entre 0.55 y 0.90 . La fiabilidad de 0.55 es para la meta de tarea y la fiabilidad de 0.90 para la meta de autofrustración.

Esta fiabilidad más baja en la meta de tarea, concuerda con los resultados de la muestra, con una media de 4.50 y una desviación típica de 0.37 . Es decir, casi toda la muestra dice tener metas de tarea en su aprendizajes. Por tanto estos datos son acogidos con cautela debido a la fiabilidad de 0.55 en meta de tarea, y nos llevan a la opinión de que cuando se cumplimentan cuestionarios en el aula delante del profesor que habi-

Tabla 3. Correlación de Pearson entre las metas de Skaalvik

\begin{tabular}{|c|c|c|c|c|c|}
\hline & & Met.Tarea & Met.Autoensalz. & Met.Autofrust. & Met.Evit. \\
\hline \multirow[t]{3}{*}{ Met. Tarea } & Correlación de Pearson & 1 & $0.20(*)$ & 0.11 & -0.10 \\
\hline & Sig (bilateral) & & 0.04 & 0.25 & 0.92 \\
\hline & $\mathrm{N}$ & 103 & 103 & 103 & 103 \\
\hline \multirow[t]{3}{*}{ Met. Autoensalz. } & Correlación de Pearson & $0.20(*)$ & 1 & 0.10 & 0.07 \\
\hline & Sig (bilateral) & 0.768 & & 0.30 & 0.40 \\
\hline & $\mathrm{N}$ & 103 & 103 & 103 & 103 \\
\hline \multirow[t]{3}{*}{ Met. Autofrust. } & Correlación de Pearson & 0.11 & 0.10 & 1 & 0.15 \\
\hline & Sig (bilateral) & 0.25 & 0.30 & & 0.12 \\
\hline & $\mathrm{N}$ & 103 & 103 & 103 & 103 \\
\hline \multirow[t]{3}{*}{ Met. Evit. } & Correlación de Pearson & -0.10 & 0.07 & 0.15 & 1 \\
\hline & Sig (bilateral) & 0.21 & 0.44 & 0.12 & \\
\hline & $\mathrm{N}$ & 103 & 103 & 103 & 103 \\
\hline
\end{tabular}

(*) La correlación es significativa al nivel 0.05 (bilateral). 
Tabla 4. Resultados del cluster mediante el procedimiento de k-medias

\begin{tabular}{lccc}
\hline & $\begin{array}{c}\text { Conglome- } \\
\text { rado 1 }\end{array}$ & $\begin{array}{c}\text { Conglome- } \\
\text { rado 2 }\end{array}$ & $\begin{array}{c}\text { Conglome- } \\
\text { rado 3 }\end{array}$ \\
\hline Meta de Tarea & 4.58 & 4.39 & 4.52 \\
Meta de Autoensalzamiento & 4.01 & 2.19 & 2.77 \\
Meta de Autofrustración & 2.45 & 1.68 & 3.97 \\
Meta de Evitación del trabajo & 2.70 & 2.48 & 2.92 \\
Número de casos & 34 & 31 & 37 \\
\hline
\end{tabular}

tualmente imparte la asignatura los estudiantes siempre reflejan como finalidad de sus aprendizajes el conocimiento en sí mismo aunque luego su motivación sea realmente otra. Si además nos fijamos en la meta de evitación del trabajo, meta peor vista en el proceso enseñanza-aprendizaje, obtenemos una media de 2.40 (la menos elegida por los estudiantes) y la fiabilidad es de 0.69 , la segunda más baja.

El análisis factorial realizado sobre la muestra obtiene 4 factores que coinciden con las cuatro metas propuestas por Skaalvik: la meta de tarea, la meta de autoensalzamiento del ego, la meta de autofrustración del ego y la meta de evitación del trabajo. Entre las metas la correlación es significativa entre la meta de tarea y la meta de autoensalzamiento del ego.

En el análisis de cluster se han obtenido tres grupos de estudiantes. Casi todos los alumnos de la muestra dicen tener siempre como motivación una alta meta de tarea. El primer grupo además, combina dentro de sus motivaciones valores altos de meta de rendimiento de demostración de las propias capacidades ante los demás y valores medios de evitar ser enjuiciado por los demás y de realización del trabajo con el menor esfuerzo posible. El segundo grupo lo componen los estudiantes que parecen disfrutar más del conocimiento y del solo hecho de aprender en sí mismo, con alta meta de tarea y bajos valores de las demás. Y por último, el tercer grupo presenta además de una alta meta de tarea, una orientación alta a evitar los juicios negativos de los otros estudiantes, con valores medios de demostración de las propias capacidades ante los demás y de realización del trabajo con el menor esfuerzo posible.

Los alumnos de nuestra muestra además, adoptan múltiples metas en sus estudios, predominando la meta de tarea entre los estudiantes. Las diferencias de estos resultados con respecto a anteriores investigaciones pueden ser debidas las peculiaridades de la muestra (universidad privada) o a las características de los estudios realizados: Diplomatura de Enfermería, que tradicionalmente se ha asociado a una carrera de elección vocacional.

Lo que es evidente, es la utilización por parte de los alumnos de metas de tarea junto a metas de rendimiento, como ponen de manifiesto dos de los grupos obtenidos, y también la utilización de metas de evitación del trabajo.

Por ello las metas elegidas por los estudiantes no son mutuamente excluyentes sino que se asocian unas con otras, y determinan en gran forma las estrategias utilizadas por los alumnos en sus aprendizajes (Suárez, González, Abalde, Valle, Rodríguez y Piñeiro 2001), posible cuestión para analizar en una investigación posterior.

La utilización de metas de tarea y de rendimiento por parte de los estudiantes, es importante al dotarles de más versatilidad a la hora de enfocar sus estudios, es evidente que en algunas situaciones las tareas son aburridas y necesitan motivación extrínseca para realizarse, por tanto el contexto y la naturaleza de la tarea son determinantes.

\section{Discusión y propuestas para la práctica educativa}

La teoría de metas proporciona gran cantidad de información sobre la motivación del alumno (Suárez y Fernández, 2004). Los estudiantes deben conocer sus razones, creencias y las emociones relacionadas con el estudio, de tal manera que puedan mantener su compromiso en la tarea y evitar la impulsividad en las actuaciones y en las tomas de decisiones (Núñez, 2008).

Por tanto, las aplicaciones de la investigación acerca de la teoría de metas aportan:

- Una línea de investigación más realista, que implica diferentes tipos de metas no como motivos antagónicos sino como objetivos complementarios a los que el estudiante recurre en función de sus intereses personales y en función de las demandas situacionales (Núñez, 2008).

- Nuevas perspectivas para el profesor que puede considerar otros motivos que no sean los de aprendizaje, como el interés en la aprobación de los demás o los premios o recompensas externas para enseñar actividades poco interesantes.

- La posibilidad de relacionar la motivación a través de las metas con las estrategias de aprendizaje que utilizan los estudiantes dependiendo de las tareas y el contexto en que se encuentren.

En definitiva, la capacidad de desarrollar la autorregulación del aprendizaje, que no se considera como mero traspaso de los conocimientos profesor-alumno, sino que requiere la construcción de los mismos por parte del propio individuo (Suárez y Fernández, 2004).

In the case of the processes of motivation, it is estab- 


\section{Extended Summary}

lished that behavior is always goal-oriented. These goals play an important part in the motivational process, as they define its content and direction (Suárez and Fernández, 2004). Skaalvik (1997), proposes four types of academic goals: task goals, self-enhancing goals, self-defeating goals, and work avoidance goals. Task goals are defined as "the desire to learn", without thinking about external rewards. There are two ego-oriented goals, or of performance: self-enhancing goals in which the person wants to demonstrate his/her capabilities to others, and self-defeating goals, in which the person is not interested in negative judgements. The final goal is the goal of work avoidance, where the goal is to do the tasks with the least possible effort.

Traditionally, these goals have been considered mutually exlusive, so that the student who directed their efforts in a task-oriented way, did not yield to another goal, but studies such as that by Suarez, Gonzalez \& Valle (2001), carried out with a sample of university students in Spain, show that students adopt multiple goals in their studies and they also relate to certain self-regulatory learning strategies.

The aim of this study was to determine the main features of academic goals in a sample of university students in their second year of studies for a nursing diploma at a private university. The following were also studied:

- The reliability and construct validity of the instrument.

- The predominant type of goals used by students.

- The type of students according to their goals.

The research is mainly descriptive in nature, focusing on validating the instrument, the questionnaire by Skaalvik (1997), and analysis of the four goals set out by Skaalvik (task goal, self-enhancing, self-defeating, and work avoidance goals).

The sample consisted of 103 students in the $2^{\text {nd }}$ year of their studies for a nursing diploma at the private University 'Alfonso X'. The campus is located in Villanueva de la Cañada (Madrid). Of the sample, 16 are men and 87 women, aged between 20 and 30. The sample was selected from students attending classes for the Mental Health Nursing course. They were from four different groups, two with classes in the morning and two in the afternoon.

The instrument used for data collection is the Skaalvik goals questionnaire (1997). The application test was conducted at a single point in time separately in each of the groups (2202, 2203, 2204 and 2205), each group in their classroom, two groups during the morning and two in the afternoon.

First, we conducted a factor analysis of the questionnaire structure, using a principal components analysis with varimax rotation. The number of factors was specified (4) to check whether our results were consistent with previous research which had established the exis- tence of four factors which group the items on the questionnaire, coinciding with the four types of goals. Second, we analyzed the level of goals, with an analysis of reliability through Cronbach's of the questionnaire in its entirety. The means and standard deviations of each goal were then studied at a descriptive level, as well as the correlations. Finally, an analysis of cluster targets was carried out, in order to study how students are grouped according to their adoption of multiple goals.

Results and conclusion. The Skaalvik goals questionnaire gives a reliability of 0.72 , thus acceptable, and each goal has a reliability of between 0.55 and 0.90 . There is a reliability of 0.55 for task goal and a reliability of 0.90 for self-defeating. This lower reliability in the case of task-oriented is consistent with the results of the sample, with an average of 4.50 and a standard deviation of 0.37 . This means that almost the entire sample said they have goals in their learning task and these data are to be taken with caution, with a reliability of 0.55 for Target Task. The data lead us to the view that when questionnaires are completed in the classroom in the presence of the teacher who usually teaches the subject, students always express knowledge in itself as the objective of their learning, even if their motivation is really another. Similarly, if we take the goal of work avoidance, the goal most negatively percieved in the teaching-learning process, we get an average of 2.40 (the least chosen by students), and the reliability is 0.69 , the second lowest.

The factor analysis carried out on the sample obtained 4 factors that coincide with the four goals set by Skaalvik: target task, self-enhancing goal, selfdefeating goal, and work avoidance goal.

Within each goal the correlations between items were significant and high, except for target task goals, and the correlation between goals is significant between task goal and self-enhancing goal

In the cluster analysis, three groups of students were found. Almost all students in the sample say they always have high goal task motivation. The first group also combines within their motivations high values of yield goal of demonstrating their own abilities to others and values avoiding judgement by others, and the work is done with the least possible effort.

The second group consists of students who seem to enjoy knowledge more and learn the simple fact in itself, with high target task and low values in other types. Finally, the third group also shows a high goal task, high orientation to avoid negative judgments by other students, with mean demonstration of abilities to others and the work being done with the least effort possible. The goal of high values of the three task groups could be related to vocational aspects of science degrees in health, in this case nursing.

Finally, this research on goal theory provides:

- A more realistic line of inquiry, which involves 
different types of goals as reasons that are not antagonistic, but rather complementary objectives which students use according to their personal interests and according to situational demands (Nuñez, 2008).

- New perspectives for the teacher who may consider other motives than learning, such as an interest in the approval of others, or extrinsic rewards, in the teaching of uninteresting activities.

The possibility of linking motivation through goals with the learning strategies used by students depending on the tasks and the context.

\section{Referencias}

Beltrán, J. (1998). Claves psicológicas para la motivación y el rendimiento académico. En M. costa (Coord.), Creatividad, motivación y rendimiento académico. Málaga: Aljibe.
González-Pienda, J. A., González, R., Núñez, J. C. y Valle, A. (2002). Manual de psicología de la educación. Madrid: Psicología Pirámide.

Núñez, J. C. (2008). Aportaciones de la psicología educativa al proceso enseñanza-aprendizaje. Oviedo: FOCAD.

Skaalvik, E. M. (1997). Self-enchancing and self-defeating ego orientation: Relations with task and avoidance orientation, achievement, self-perceptions, and anxiety. Journal of Educational Psychology, 89, 71-81.

Suárez, J. M. y Fernández, A. P. (2004). El aprendizaje autorregulado: variables estratégicas, motivacionales, evaluación e intervención. Madrid: UNED.

Suárez, J. M., González, R., Abalde, E., Valle, A., Rodríguez, S. y Piñeiro, I. (2001). La adopción de múltiples metas y utilización de estrategias cogniticas y autorreguladoras. Bordon, 53, 129-139.

Suárez, J. M., González, R. y Valle, A. (2001). Multiple goal pursir and its relation to cognitive, self-regulatory, and motivational strategies. British Journal of Educational Psychology, 71, 561-572.

Manuscrito recibido: 18/07/2011

Revisión recibida: 26/09/2011

Manuscrito aceptado: 10/10/2011 